\title{
Toward an understanding of the pathophysiology of clear cell carcinoma of the ovary (Review)
}

\author{
CHIHARU UEKURI, HIROSHI SHIGETOMI, SUMIRE ONO, \\ YOSHIKAZU SASAKI, MIYUKI MATSUURA and HIROSHI KOBAYASHI
}

Department of Obstetrics and Gynecology, Nara Medical University, Kashihara, Nara 634-8522, Japan

Received January 31, 2013; Accepted July 29, 2013

DOI: $10.3892 / \mathrm{ol} .2013 .1550$

\begin{abstract}
Endometriosis-associated ovarian cancers demonstrate substantial morphological and genetic diversity. The transcription factor, hepatocyte nuclear factor (HNF)-1 $\beta$, may be one of several key genes involved in the identity of ovarian clear cell carcinoma (CCC). The present study reviews a considerably expanded set of HNF-1 $\beta$-associated genes and proteins that determine the pathophysiology of CCC. The current literature was reviewed by searching MEDLINE/PubMed. Functional interpretations of gene expression profiling in CCC are provided. Several important CCC-related genes overlap with those known to be regulated by the upregulation of HNF-1 $\beta$ expression, along with a lack of estrogen receptor (ER) expression. Furthermore, the genetic expression pattern in CCC resembles that of the Arias-Stella reaction, decidualization and placentation. $\mathrm{HNF}-1 \beta$ regulates a subset of progesterone target genes. HNF-1 $\beta$ may also act as a modulator of female reproduction, playing a role in endometrial regeneration, differentiation, decidualization, glycogen synthesis, detoxification, cell cycle regulation, implantation, uterine receptivity and a successful pregnancy. In conclusion, the present study focused on reviewing the aberrant expression of CCC-specific genes and provided an update on the pathological implications and molecular functions of well-characterized CCC-specific genes.
\end{abstract}

\section{Contents}

1. Introduction

2. Review of the literature

3. CCC-specific genetic signaling circuitries

4. CCC-specific epigenetic signaling circuitries

5. HNF-1 $\beta$ and its related genes

Correspondence to: Professor Hiroshi Kobayashi, Department of Obstetrics and Gynecology, Nara Medical University, 840 Shijo-cho, Kashihara, Nara 634-8522, Japan

E-mail: hirokoba@naramed-u.ac.jp

Key words: clear cell carcinoma, ovarian cancer, endometriosis, HNF-1 $\beta$, estrogen receptor
6. Summary

\section{Introduction}

Epithelial ovarian cancers are a heterogeneous group with varying pathologies, and are broadly categorized into serous, mucinous, endometrioid and clear cell histotypes. These histological subtypes demonstrate morphological features that resemble the Müllerian duct-derived epithelial cells, which are embryologically derived from the fallopian tubes, endocervix, endometrium and gestational endometrium, respectively. Epithelial ovarian cancer is generally managed as a single entity, and treated with a combination of maximal cytoreductive surgery and taxane/platinum-based chemotherapy (1). Randomized clinical trials have allowed us to develop a number of successful therapeutic strategies using paclitaxel and carboplatin for serous cancer, which is the most prevalent histotype (2). However, the current standard treatments applied to high-grade serous cancer are ineffective for clear cell carcinoma (CCC) (1). CCC is more likely to be chemoresistant than high-grade serous cancer. The differential expression of chemoresistance-related genes has been observed in various histological types of ovarian cancer (1).

The intrinsic chemoresistance of CCC appears to be related to an increase in the detoxification of the drug within the cell or an increase in cell cycle arrest during the DNA damage response (3). Previous studies have demonstrated that the majority of the CCC-specific genes display a significant change in expression upon exposure to an oxidative stress microenvironment (3-5). Several studies have summarized the clinicopathological features, phenotypic and genomic changes in CCC and provided mechanistic interpretations of the gene expression changes, including chemoresistance, cell cycle regulation, hormone independency, detoxification, glycogen synthesis and chromosomal instability $(3,6,7)$.

Endometriosis-associated neoplasms include CCC, endometrioid adenocarcinoma, serous borderline tumors, Müllerian mucinous borderline tumors, adenosarcoma and endometrial stromal sarcoma (8). Substantial histopathological data provide evidence that CCC and endometrioid adenocarcinoma are the most common histologies in ovarian cancer patients who have associated endometriosis, also known as endometriosis-associated ovarian cancer. Abundant iron-mediated oxidative stress 
occurs due to repeated hemorrhage in endometriosis, prior to this compound oxidatively modifying host genomic DNA stability, which is a significant factor that accelerates carcinogenesis (9). Thus, the pathogenesis of endometriosis-associated ovarian carcinogenesis may be closely associated with iron overload. Iron overload-related diseases, including hemochromatosis, chronic viral hepatitis, asbestosis and endometriosis, may lead to the development of hepatocellular carcinoma, mesothelioma or ovarian cancer (10). Such microenvironments may play a role in carcinogenesis. Nevertheless, CCC and endometrioid adenocarcinoma have distinct clinicopathological characteristics and molecular phenotypes $(11,12)$. The present study reviews a considerably expanded functional set of CCC-specific genes and discusses recent insights into the pathophysiology of CCC.

\section{Review of the literature}

A comprehensive review of the literature was conducted in order to investigate the molecular basis of the CCC-specific genes. A MEDLINE search of the literature was performed using the key words 'endometriosis', 'ovarian cancer', 'clear cell carcinoma', 'estrogen receptor', 'Arias-Stella reaction' and 'HNF-1 $\beta$ '. English-language publications in PubMed and references from relevant articles published between 2005 and 2012 were analyzed. References in the studies identified were also searched.

\section{CCC-specific genetic signaling circuitries}

Several genome-wide gene expression analyses have led to the identification of sets of CCC-specific genes and their regulated targets, and highlighted a complex circuitry responsible for CCC development, maintenance and progression (5). Two characteristics define CCC cells, hormone independency and HNF-1 $\beta$ overexpression. A previous study on the immunophenotype of CCC cells showed that the tumor cells are positive for HNF-1 $\beta$ and insulin-like growth factor binding protein-1 (IGFBP-1), but negative for estrogen receptor (ER), progesterone receptor (PR), Wilms tumor 1 (WT1) and p53 (13).

The expression of the hormone receptors, ER and PR, is more elevated in cells of endometrioid-type than in those of non-endometrioid origin (14). ER and PR expression may be a significant event in the progression of endometrioid carcinoma, which develops from endometrial hyperplasia with the risks of unopposed estrogen signaling in the endometriotic epithelium. Notably, CCC has shown negative or weak staining for ER- $\alpha$ and PR (13). The frequent loss of estrogen function may be a turning point in CCC progression and aggressiveness. DNA methylation of the promoter region, histone deacetylation, chromatin remodeling and ubiquitination may negatively regulate ER expression in CCC (11).

Among epithelial ovarian cancers, the majority of CCC cases showed the expression of the HNF-1 $\beta$ gene, whereas non-CCC tumors hardly expressed this gene (15). The CCC-specific genes are in extremely close linkage with HNF-1 $\beta$ and its target genes. HNF-1 $\beta$ may be a central player in the differentiation into CCC-specific lineages from endometriosis (4). There is a significant overlap between the HNF-1 $\beta$-related genes and those associated with chemoresistance, detoxification and glycogen synthesis, with the conservation of the biological features of CCC $(3,7,11,12)$. HNF-1 $\beta$ plays a role in the cellular stress response in CCC through its regulation of cytoprotective genes (3). Despite recent studies, our understanding of the HNF-1 $\beta$-associated molecular mechanisms and functional players in CCC remains limited.

To further elucidate the biological pathway network, the present study reviewed extensive functional studies of CCC-specific genes and proteins at the transcriptome and proteome levels. The CCC-specific genes were classified into several groups based on assorted physiological functions (Fig. 1). The differentially expressed genes are mainly involved in several functional groups, including those of decidualization, placentation and successful pregnancy, the Arial-Stella reaction, differentiation, glycogen synthesis, detoxification, chemoresistance, endometriosis development and ion channels. Progesterone is required to develop and maintain the decidualized phenotype of the endometrium and plays a role in epithelial-mesenchymal communication (16). The process of the differentiation of endometrial stromal cells into secretory cells, known as decidualization, is a prerequisite for the successful implantation, progression and maintenance of a pregnancy. The placenta is critical for nourishing the fetus in order to meet the requirements of the growing fetus throughout pregnancy, and also produces metabolizing enzymes that detoxify foreign chemicals. Ion channels play important roles in the feto-placental vasculature and are essential for electrolyte absorption and replacement of essential amino acids (17). These data allow us to speculate that the CCC-specific genes stimulate the secretory functions of the endometrium that are required for differentiation and implantation, placentation, and pregnancy recognition signaling, and are essential for fetal development.

Arias-Stella reaction. Epigenetic, genetic, morphological and functional changes occurring within the endometrium during the menstrual cycle are orchestrated under the influence of sequential exposure to the cyclic expression of the ovarian steroids, estradiol and progesterone. These changes are required for the development of uterine receptivity, the disintegration of the decidualized endometrium and the subsequent repair and fine remodeling for tissue regeneration, differentiation and placentation. Two independent ERs, ER- $\alpha$ and ER- $\beta$, have distinctive cellular distribution patterns in the reproductive organs. ER- $\beta$ predominantly inhibits the activity of ER- $\alpha$. The expression of ER- $\alpha$ has been identified as lower during the secretory phase compared with the proliferative phase, and significantly lower in the first-trimester decidua (18). In contrast to ER- $\alpha$, ER- $\beta$ was observed to be expressed in all compartments of the decidual tissues. The PR gene produces two isoforms, PRA and PRB, which are mainly localized to the nuclei of stromal cells. PR was expressed at a lower level in the secretory endometrium than in the proliferative endometrium (18).

Although ER expression in endometriosis is variable, several studies have reported higher levels of ER- $\beta$ and lower levels of ER- $\alpha$ (19). The expression of PR, particularly the PR-B isoform, is decreased, demonstrating that the resistance of endometriotic tissue to progesterone, so-called progesterone resistance, is commonly observed in females with 


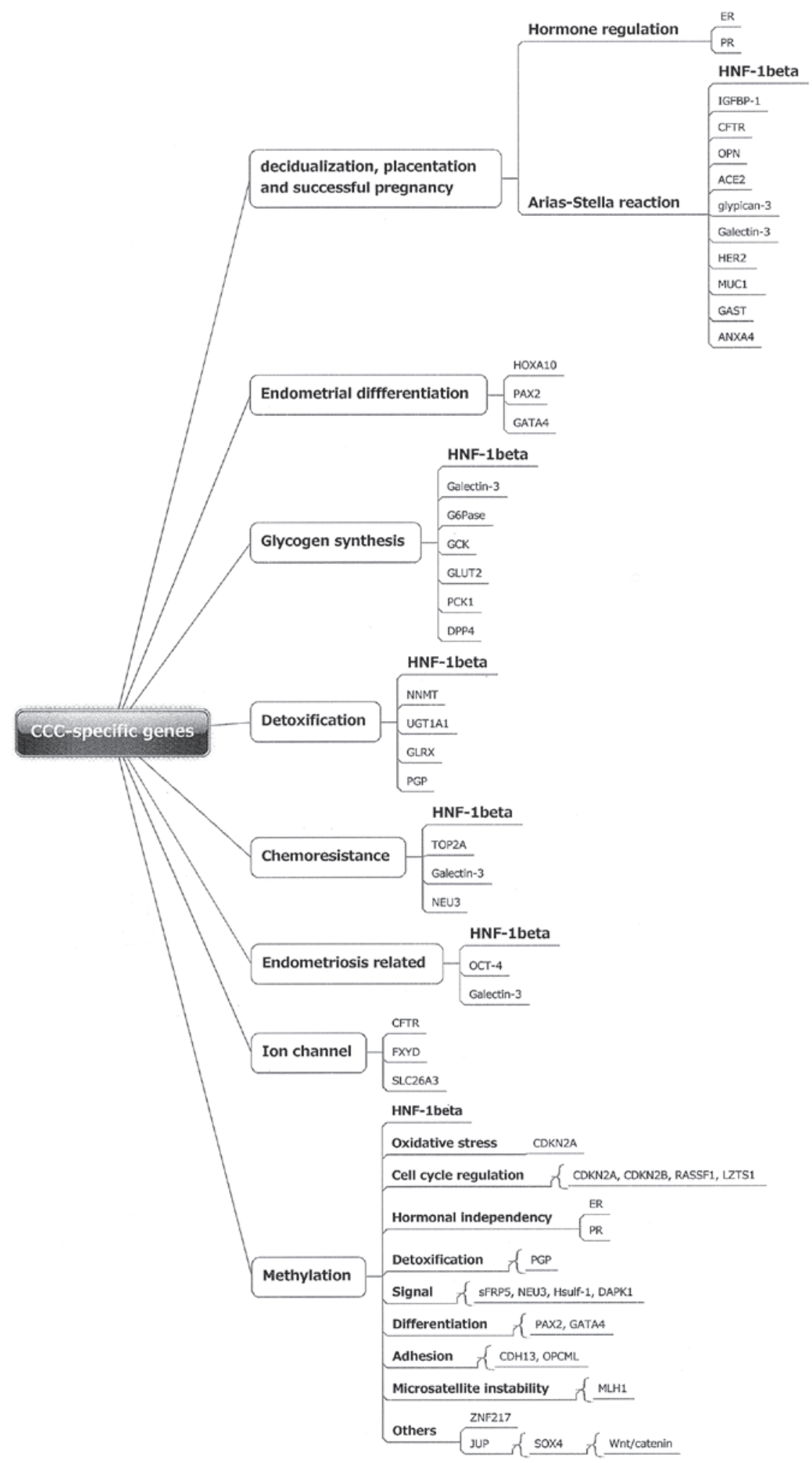

Figure 1. Regulation of gene expression associated with molecular functions and biological processes in clear cell carcinoma (CCC). Gene expression was analyzed with respect to genome-wide gene expression profiling and gene ontology groups. The gene tree starts with the top term 'CCC-specific genes' and then splits out into more specific functional terms. Each box contains the name of the process. The CCC-specific genes were classified into diverse functional categories and signalling pathways, including decidualization, endometrial differentiation, glycogen synthesis, detoxification, chemoresistance and ion channels.

endometriosis (19). The endometriotic ER and PR expression pattern resembles that of the eutopic secretory endometrium. Furthermore, in a study on endometrioid adenocarcinoma, ER- $\alpha$ expression increased with malignant transformation. By contrast, a gradual reduction in ER- $\alpha$ and PR expression was observed with malignant transformation from endometriosis to atypical endometriosis to CCC (20). There is a marked tendency for the disappearance of these receptors in association with the malignant transformation of endometriosis into CCC. 
The Arias-Stella reaction is a hormone-induced hyperplastic change of the endometrial epithelium that is associated with cellular atypia, which may be the result of the regeneration and proliferation activities of the endometrial or endometriotic glands (21). The changes in this reaction, a common and universally recognized feature of the gestational endometrium, have been frequently observed in abortion curettage specimens (21). The reaction was also observed in the cytoplasm of stromal cells undergoing decidual change in certain patients treated with exogenous hormones, such as progestin (21). Endometriosis infrequently shows decidual changes and a Arias-Stella reaction, suggesting the possibility of a metaplastic origin from the secondary Müllerian system (22,23). Morphological and phenotypic features of the CCC cells resemble those of cells undergoing the Arias-Stella reaction (24). The Arias-Stella reaction and $\mathrm{CCC}$ often show prominent hobnail features and clear cell changes. The etiology for the cytoplasmic clearing observed in these cells includes the accumulation of intracellular glycogen. Phenotypical homology may emphasize common ancestry through the genetic continuity. However, ER and PR expression has shown a marked difference between endometrial epithelial cells, corresponding to the Arias-Stella reaction (positive staining), and CCC (often appears negative), indicating that clear cells in the two entities are phenotypically similar but hormonally distinct. CCC cells are able to exploit a progression mechanism via hormone-independent signaling pathways $(11,21)$.

\section{Decidualization, placentation and a successful pregnancy}

The unique gene families discussed in this section may be closely related to decidualization, placentation and a successful pregnancy. These proteins are expressed at a higher level in the secretory endometrium than in the proliferative endometrium. The insight provided by this review may illuminate how the hormone-independent state is established and regulated.

Osteopontin (OPN), also known as secreted phosphoprotein 1. OPN is a glycoprotein of the extracellular matrix that also acts as a chemokine. OPN is most likely a direct target gene of HNF-1 $\beta$, as it contains functional HNF1 binding sites in its promoter region (25). OPN is expressed in the cyclic endometrium, decidual stromal cells and natural killer cells, and higher expression has been detected in the later gestational phase compared with the early gestational phase. OPN expression was shown to be regulated by progesterone (25). The involvement of OPN has been proposed for maintaining successful pregnancy. Furthermore, OPN was shown to be overexpressed in the late secretory endometrium of females with endometriosis (25). This glycoprotein has been shown to mediate cellular invasion and to contribute to tumorigenesis in several types of cancer. OPN may be involved in the pathogenesis of endometriosis and endometriosis-associated CCC, and its regulation may have a crucial role in CCC therapy (26).

Angiotensin converting enzyme 2 (ACE2). The ACE2 gene contains functional HNF1 binding sites and its overexpression has been observed in CCC. ACE2 metabolizes Angiotensin II, a vasoconstrictor, to Angiotensin (1-7), a vasodilator. This enzyme stimulates endothelial cell migration, capillary formation and angiogenesis, and attenuates angiotensin II-induced reactive oxygen species (ROS) production (27). ACE2 expression has been shown to be elevated in the secretory phase of endometrial epithelial cells and early gestational trophoblasts. One of the first signs of implantation is an increase in endometrial vascular permeability and angiogenesis (27). Thus, ACE2 may act as a local autocrine/paracrine regulator of angiogenesis, vascular permeability and growth during decidualization and implantation (?).

Galectin-3 and lectin, galactoside-binding, soluble 3 (LGALS3). Galectin-3 protein has been immunohistochemically observed in $59.7 \%$ of CCC cases (28). It has been implicated in cell cycle control, adhesion, migration, invasion, angiogenesis and tumor development through the activation of the Wnt signaling pathway. The overexpression of galectin-3 in CCC may contribute to cisplatin resistance (29). Galectin-3 has been shown to be expressed in endometrial epithelial cells during the secretory phase of the menstrual cycle, and to regulate cell-cell adhesion by interacting with integrin- $\beta 3$ during embryo implantation (30). 17 $\beta$-estradiol, progesterone and human chorionic gonadotropin induced the expression of galectin-3 (30). Galectin-3 also stimulates the expression of phosphorylated glycogen synthase kinase-3 $\beta$ (GSK $3 \beta$ ) and regulates glycogen synthesis. Furthermore, galectin-3 expression has been observed to be higher in endometriosis than in the eutopic endometrium, indicating that galectin-3 has a potential role in the development of endometriosis and subsequently, in CCC (31).

Glypican-3 (GPC3). The expression of GPC3, a heparan sulfate proteoglycan, has been observed in $44 \%$ of CCC cases, while rarely being observed in other histological subtypes (32). GPC3 regulates cellular growth by interacting with a variety of morphogenic or growth factors, such as Wnt, fibroblast growth factor (FGF) 2 and bone morphogenic protein (BMP). The expression of GPC3 has been identified in the endometrial epithelium in the gestational period (32). The differentiated syncytiotrophoblast cells derived from the placenta expressed GPC3 mRNA and protein.

Mucin 1, cell surface associated (MUC1). The cancer-associated aberrantly sialylated MUC1 glycoprotein specifically expressed on CCC plays a role in tumorigenicity, survival under anoikis conditions and malignant behavior (33). Progesterone stimulates the expression of MUC1. MUC1 has been observed to be expressed in endometriosis (34). The endometrium of fertile females also expresses MUC1, which carries selectin ligands recognized by the human blastocyst. MUC1 expression may be required at implantation sites to permit embryo attachment and implantation (34,35).

Annexin A4 (ANXA4). ANXA4 expression is elevated in $\mathrm{CCC}$ and enhances cancer cell chemoresistance (36). This protein belongs to a family of calcium-dependent phospholipid-binding proteins and is involved in ion channel regulation, exocytosis and signal transduction. Studies have shown that ANXA4 mRNA is significantly upregulated during mid-to-late-secretory phases compared with proliferative phases, and that progesterone, but not estrogen, increased the expression of ANXA4 mRNA and protein (37). The lower expression of ANXA4 during implantation in infertile patients with endometriosis may be associated with the decrease of endometrial receptivity (38).

Insulin-like growth factor binding protein-1 (IGFBP-1). IGFBP-1 is a specific marker for CCC (39). The expression of IGFBP-1 may be more specific than that of HNF-1 $\beta$. The 
specific expression of IGFBP-1 has also been confirmed in the secretory endometrium, the decidua of the placenta and the Arias-Stella glands (39). Progesterone induces the expression of IGFBP-1. IGFBP-1 is downregulated in endometriosis via reduced levels of forkhead box $\mathrm{O} 1$ (FOXO1) and homeobox A10 (HOXA10), upstream target genes of IGFBP-1.

Human epidermal growth factor receptor 2 (HER2), also known as v-erb-b2 erythroblastic leukemia viral oncogene homolog 2. HER2/neu-positivity in CCC has been observed to be significantly higher than in other ovarian cancers (40). This oncogene showed the highest level of expression during the early secretory phase and in the placenta. HER2 plays fundamental roles in embryogenesis, development, proliferation and differentiation.

Octamer-binding protein 4 (OCT-4), also known as POU class 5 homeobox 1. CCC shows positive staining (28\%) for the stem cell marker OCT-4, which is key in stem cell identity and reprogramming (41). The transcription factor OCT-4 is one of the most likely markers for endometrial stem cells. OCT-4 plays a role in embryonic development, particularly during early embryogenesis. Endometriotic cells express OCT-4, indicating that the ectopic endometrium in endometriosis has a stem cell origin and may explain the possible progression to ovarian cancer (42).

\section{Endometrial differentiation}

The proteins that increase between the early and mid-luteal phases may play direct roles in embryo-uterine interactions during the implantation process (43). These implantation-related genes include HOXA10, MUC1, leukemia inhibitory factor (LIF), glycodelin, matrix metalloproteinase (MMP), tissue inhibitors of matrix metalloproteinase (TIMP) and E-cadherin. The majority of these genes are overexpressed in $\mathrm{CCC}$.

HOXA10. In a study by Li et al (44) HOXA10 was not expressed in the normal ovarian epithelium, endometriosis and ovarian serous carcinomas samples, while $68.9 \%$ of the CCC samples were positive for the expression of HOXA10. HOXA10 is a DNA-binding transcription factor that regulates gene expression, morphogenesis and differentiation. This gene regulates endometrial receptivity and its expression is decreased in females with endometriosis. HOXA10 is expressed cyclically during the menstrual cycle in the endometrium, under the influence of sex steroids, with the highest expression during the implantation window (45).

\section{Glycogen synthesis}

Glycogen accumulation operates as an energy source, enabling cell growth even under conditions of oxidative stress. Genes associated with glycogen synthesis are related to a mechanism of cisplatin (CDDP)-resistance in CCC $(3,46)$.

Glucose-6-phosphatase (G6Pase). G6Pase catalyzes the hydrolysis of D-glucose 6-phosphate to D-glucose (47). The endometrium and placenta produce glucose via G6Pase. The key metabolic change that occurs during the implantation period is the rise in endometrial glycogen content. Glycogen synthesis is regulated by progesterone (47).

Glucokinase (GCK), also known as hexokinase 4. This protein regulates glycerol uptake and is associated with glycogen synthesis (http://www.ncbi.nlm.nih.gov/gene/2645).
Glucose transporter type 2 (GLUT2). GLUT isoforms, of which 13 have been identified to date, mediate facilitated glucose transport. GLUT2 expression increases in the endometrium throughout the secretory phase and in the decidua (48).

Phosphoenolpyruvate carboxykinase (PCK1). PCK1 regulates gluconeogenesis. PCK1 forms phosphoenolpyruvate from oxaloacetate and its expression is regulated by insulin (http:// www.ncbi.nlm.nih.gov/gene/5105).

Dipeptidyl peptidase 4 (DPP4), also known as CD26. DPP4, also known as CD26, is identical to adenosine deaminase complexing protein-2 (http://www.ncbi.nlm.nih. gov/gene/1803). DPP4 inactivates insulin-sensing incretin hormones, such as glucagon-like peptide 1 (GLP-1) and glucose-dependent insulintropic polypeptide (GIP) (49).

\section{Detoxification}

The diagnosis of endometriosis is based on the direct or indirect evidence of cyclic bleeding in ovarian tumors. Persistent iron-induced free radicals induced by endometriosis-dependent hemorrhage may be associated with carcinogenesis. The HNF-1 $\beta$-related detoxification enzymes are crucial for protection against oxidative stress. Genes associated with detoxification are also related to a mechanism of CDDP-resistance in CCC (46).

UDP-glycosyltransferase 1 family polypeptide AI (UGT1A1). UGT1A1 is an enzyme of the glucuronidation pathway that plays a critical role in the detoxification that transforms small lipophilic molecules, including estrogen, bilirubin, hormones and drugs, into water-soluble, excretable metabolites. Menstruation and hemorrhage in endometriosis are biochemically active environments known to undergo potent oxidizing reactions. Iron and bilirubin mediate the generation of ROS via the Fenton reaction. If unconjugated or uncontrolled, they may lead to oxidative DNA and protein damage. UGT1A1 is also involved in controlling the toxicity of the chemotherapeutic agent irinotecan. The presence of UGT1A1*28 may result in an increased risk of ovarian cancer (50).

Glutaredoxin (GLRX). GLRX is important for the antioxidant defense and for the regulation of the cellular redox state. This protein protects cells from $\mathrm{H}_{2} \mathrm{O}_{2}$-induced apoptosis by regulating the redox state. GLRX plays a critical role in the detoxification of iron-induced free radicals in CCC (46).

Nicotinamide $N$-methyltransferase (NNMT). NNMT catalyzes the $N$-methylation of nicotinamide, pyridines and other structural analogs by using $S$-adenosylmethionine as a methyl donor, playing a pivotal role in the biotransformation and detoxification of numerous xenobiotics. This gene contributes to MMP-2 expression, which induces cell invasiveness via the PI3K-AKT pathway, indicating NNMT as a novel invasion-related gene (51). This protein is most likely involved in embryo-implantation mechanisms. NNMT is also one of the genes upregulated in endometrial stromal cells in response to macrophage activation in endometriosis.

\section{Ion channels}

The absorption of uterine cavity fluid in early pregnancy results in the closure of the lumen and allows blastocysts to make contact with the luminal epithelium (52). Fluid absorption peaks at the time of implantation through progesterone action. The expression patterns of the CCC-specific gene subsets 
resemble those of the ion transporting proteins involved in fluid absorption.

Cystic fibrosis transmembrane conductance regulator (CFTR), also known as ATP-binding cassette sub-family $C$, member 7 (ABCC7). The CFTR gene encodes a member of the ATP-binding cassette $(\mathrm{ABC})$ transporter superfamily. CFTR is involved in sodium and chloride absorption within the uterus. Progesterone stimulates amiloride-sensitive fluid absorption. Mutations in this gene are associated with the autosomal recessive disorder cystic fibrosis. HNF-1 interacts with multiple elements across the CFTR locus (53). CFTR appears to be overexpressed in endometriosis and CCC.

FXYD domain-containing ion transport regulator 2 (FXYD2). FXYD2 is the sodium and potassium-transporting ATPase subunit $\gamma$ and is expressed in the uterine endometrium. HNF-1 $\beta$ binding sites have been detected in the FXYD2 gene (54). Mutations in this gene have been associated with renal hypomagnesemia.

Solute carrier family 26, member 3 (SLC26A3). SLC26A3 functions to transport chloride ions across the cell membrane in exchange for bicarbonate ions (55). The protein is essential for chloride absorption and is predominantly localized to the mucosa of the lower intestinal tract.

\section{Cell cycle regulation}

Genes mapping to amplified regions in CCC include 20q13.2 (harboring ZNF217), 17q12-q21.32 (harboring HER2, TOP2A, GAST, JUP and BRCA1) and 17q23.2 (PPM1D) (40). The resistance of CCC to platinum-based chemotherapy may be caused by low levels of cell proliferation (6).

Early mitotic inhibitor-1 (Emi1). Significant overexpression of Emi1 protein was present in $82 \%$ of CCC $(55,56)$. Emi1 protein is a key cell cycle regulator that promotes $\mathrm{S}$-phase and mitotic entry by inhibiting the anaphase promoting complex. The overexpression of Emil leads to tetraploidy and genomic instability.

Zinc finger protein 217 (ZNF217). ZNF217 amplification is observed in $36 \%$ of CCC, but rarely detected in serous cancer, regardless of grade (57). ZNF217 plays a role in the chemoresistance and poor prognosis in breast and ovarian cancer, possibly through the Aurora-A signaling cascade.

Topoisomerase (DNA) II a $170 k D a$ (TOP2A). TOP2A is a cell cycle regulating gene that is upregulated in chemoresistant ovarian cancer, particularly in CCC (58). TOP2 is involved in processes, including chromosome condensation, chromatid separation and the relief of torsional stress that occurs during DNA transcription and replication.

Together, all these data indicate that the CCC-specific genes significantly overlap with the genes associated with the conservation of the biological features of CCC.

\section{CCC-specific epigenetic signaling circuitries}

The changes in the DNA hypermethylation pattern is one of the mechanisms for the earliest molecular changes in carcinogenesis. In epithelial ovarian cancer, the frequently methylated genes were reported to be the cyclin-dependent kinase inhibitor 2B (CDKN2B); estrogen receptor 1 (ESR1); secreted frizzled-related protein 5 (sFRP5); cadherin 13 (CDH13, H-cadherin); Ras association (RalGDS/AF-6) domain family member 1 (RASSF1); mutL homolog 1, colon cancer, nonpolyposis type 2 (MLH1); opioid binding protein/cell adhesion molecule-like (OPCML); sulfatase 1 (Hsulf-1); GATA binding protein 4 (GATA4) and death-associated protein kinase 1 (DAPK1) genes $(59,60)$. CCC samples exhibited a higher frequency of CDKN2B, ESR1 and SFRP5 promoter hypermethylation compared with those of other histological types (Fig. 1). In addition, homozygous deletions were detected at the CDKN2A, CDKN2B and LZTS1 loci in CCC (57). Genetic events in CDKN2A and ESR1 genes are rare in endometriosis.

\section{Cell cycle regulation}

CDKN2B, also known as p15INK4B, p15, inhibits cyclindependent kinase 4, and CDKN2A, also known as PI6INK4A. The iron-mediated formation of free radicals (e.g. ROS) has been shown to be associated with carcinogenesis. Akatsuka et al identified CDKN2A, a cell cycle control gene, and $\mathrm{CDKN} 2 \mathrm{~B}$ genes in a ferric nitrilotriacetate-induced renal carcinogenesis animal model (61). The two genes may be vulnerable to ROS in endometriosis. The allelic loss of the CDKN2A gene occurs specifically at the p16 loci.

RASSF1. RASSF1 is commonly silenced by promoter hypermethylation in a variety of types of human cancer, including ovarian cancer (62). This protein interacts with the DNA repair protein, xeroderma pigmentosum, complementation group A (XPA) and inhibits the accumulation of cyclin D1, thus inducing cell cycle arrest. No epigenetic alterations were identified in RASSF1 in endometriosis samples (63).

Leucine zipper, putative tumor suppressor 1 (LZTS1). Fasciculation and elongation protein- $\zeta 1$ (FEZ1) expression is absent or markedly reduced in $38 \%$ of ovarian cancers. Homozygous deletions are detected at the LZTS1 loci at 8p22 in CCC. LZTS1 has a role in cell-cycle control by interacting with the Cdk1-cyclin B1 complex (57).

\section{Hormonal regulation}

ESR1. The hormonal receptor profile of CCC and endometriosis is characterized by the low expression of ER- $\alpha$ and PR, and by ER- $\beta$ overexpression (64). Hypomethylation at the ER- $\beta$ promoter is responsible for high ER- $\beta$ levels (19). ER- $\beta$ suppresses ER- $\alpha$ levels. An increased ER- $\beta$ to ER- $\alpha$ ratio is responsible for decreased PR expression.

\section{Detoxification}

P-glycoprotein (PGP). CCC has a lower expression of multi-drug resistance PGP than serous cancer in females (65). The proliferative endometrium has revealed no PGP expression, while the secretory and menstrual endometrium has been identified with positive staining. Progesterone increases PGP expression and function. All gestational endometria have shown positive staining for PGP in the Arias-Stella reaction and the decidua. PGP protects the fetus from exposure to xenobiotics during pregnancy (66).

\section{Signaling}

sFRP5. The sFRP5 promoter has been shown to be predominantly methylated in CCC tissues, with $64.6 \%$ in CCC compared with $13.3 \%$ in serous cancer, and $0 \%$ in endometriosis and the normal ovarian epithelium (60). sFRP5 modulates Wnt signals, which play a significant role in reproductive events. 
SFRP5 may regulate endometrial stromal cell proliferation, survival and differentiation, which is required to support the developing embryo.

NEU3 (encodes sialidase 3). Plasma membrane-associated NEU3 is expressed in the majority of CCC cases. The overexpression of NEU3 significantly enhances cell resistance to hypoxia (67).

Hsulf-1. Heparan sulfate 6-O-endosulfatases, such as HSulf-1, selectively remove 6-O-sulfate from heparan sulfate, upregulate heparin-binding growth factor signaling and confer resistance to chemotherapy-induced apoptosis. HSulf-1 inactivation in CCC is partly mediated by the loss of heterozygosity and epigenetic silencing (68). HNF-1 $\beta$ negatively regulates HSulf-1 expression.

$D A P K 1$. DAPK 1 is a positive mediator of TNF- $\alpha$ and $\gamma$-interferon-induced apoptosis via the NF- $\mathrm{B}$ signaling pathways. Collins et al reported low levels of DAPK1 expression in CCC compared with in normal samples (69).

\section{Differentiation}

Paired-box gene 2 (PAX2). PAX2 is a target of transcriptional suppression by the tumor suppressor gene, WT1, and is essential in embryonic development of Müllerian organs. Promoter hypomethylation of the transcription factor PAX2 has been identified in $75 \%$ of CCC cases (70).

GATA4. The family of zinc finger-containing GATA transcription factors is frequently lost in ovarian cancer, and this loss accounts for the dedifferentiation of the cancer cells (71). GATA4 has also been shown to be frequently lost in preneoplastic lesions, including morphologically normal inclusion cysts, epithelial hyperplasia or atypical endometriosis adjacent to malignant cells. GATA4 plays critical roles in cell lineage specification during early embryonic development and organ formation.

\section{Adhesion}

CDH13. CDH13, glutathione S-transferase- $\pi 1$ (GSTP1) and RASSF1 are frequently methylated in sporadic and BRCA1-associated ovarian cancers. $\mathrm{CDH} 13$ is a cell adherence protein and is often silenced in cancer cells. Like CCC, epigenetic alterations in $\mathrm{CDH} 13$ and $\mathrm{CDKN} 2 \mathrm{~A}$ have been observed in a silica-induced lung cancer model (72).

OPCML. OPCML is frequently inactivated by allelic loss and $\mathrm{CpG}$ island promoter methylation in epithelial ovarian cancer (73). OPCML may have an accessory role in opioid receptor function and negatively regulate a specific repertoire of receptor tyrosine kinases, including EPH receptor A2 (EPHA2), fibroblast growth factor receptor 1 (FGFR1), FGFR3, HER2 and HER4.

\section{Microsatellite instability}

MLH1. Microsatellite instability is proposed to be limited to CCC and endometrioid cancer. The epigenetic inactivation of hMLH1 is also an early event in the malignant transformation of endometriosis (74). Abnormal methylation has been detected in $\sim 10 \%$ of endometriosis cases.

\section{HNF-1 $\beta$ and its related genes}

Members of the HNF-1 protein family, HNF-1 $\alpha$ and HNF-1 $\beta$, are homeobox transcription factors involved in embryonic development and tissue-specific gene expression in several organs, including the ovaries and uterus. HNF-1 $\beta$ plays a crucial role in cell differentiation and tissue morphogenesis. HNF-1 $\beta$-knockout is embryonic lethal due to the defective differentiation of the extra-embryonic visceral endoderm (75). This transcription factor controls endoderm development. HNF-1 $\beta$ may be activated during the differentiation of embryonic endodermal stem cells.

In the kidney, HNF-1 $\beta$ is upregulated after acute kidney injury in proximal tubular cells. HNF-1 $\beta$ mutations have been associated with a variety of disorders of renal development with polycystic kidney disease (76). HNF-1 $\beta$ controls cellular proliferation and tubule formation by regulating the expression of a number of kidney-specific genes, including polycystic kidney and hepatic disease 1 (PKHD1), uromodulin (UMOD) and suppressor of cytokine signaling-3 (SOCS3) expression and the signal transducer and activator of transcription 3 (STAT3)/mitogen-activated protein kinase 1 (MAPK1, Erk) activation cascade (77).

The intestinal epithelium is a complex system characterized by continuous cell renewal, differentiation and apoptosis. In the gut, the clusters of co-regulated genes associated with HNF-1 $\beta$ are HNF-1 $\alpha, \mathrm{HNF}-4 \alpha$, FABP1 and UGT2B7 (78). HNF-1 $\beta$ is also able to control the expression of a number of intestinal target genes, including FABP1, LPH, CFTR, G6Pase and DPP4. The concerted action of HNF-1 $\alpha$ and HNF-1 $\beta$ activates the expression of Notch, SLC26A3, ATOH1 and JAG1, which act on cell fate determination, stem cell self-renewal, epithelial cell polarity, adhesion, cell division, differentiation and intestinal water absorption.

In the uterine endometrium, epithelial glandular nuclei have demonstrated no HNF-1 $\beta$ expression in the proliferative phase, with significant increases in the secretory and menstrual phases (46). The pregnant endometrial glandular cells have also been demonstrated to be uniformly and continuously positive for HNF-1 $\beta$. The majority of HNF-1 $\beta$-associated gene products co-localize to the cilium, a crucial organelle that plays a significant role in controlling proliferation and differentiation (79). The present review identifies direct and indirect target genes of HNF-1 $\beta$ and shows that this transcription factor plays a crucial role in defining cell fate and controlling terminal functions in the endoderm epithelium.

Polycystic kidney and hepatic disease 1 (PKHD1). This gene modulates calcium-dependent renal epithelial cell proliferation and differentiation. PKHD1 mutations cause autosomal recessive polycystic kidney disease (80).

Uromodulin (UMOD). UMOD in urine provides a defense against urinary tract infections (81). UMOD also acts as a constitutive inhibitor of calcium crystallization.

Suppressor of cytokine signaling 3 (SOCS3). SOCS3 has emerged as a critical attenuator of cytokine-mediated processes in a negative-feedback mechanism to hinder cytokine receptor activity, indicating a role in the suppression of tumorigenesis. The DNA-hypermethylation of SOCS genes in ovarian cancer has led to speculation that silencing of the SOCS3 gene may promote the oncogenic transformation of epithelial tissues (82). In the human endometrium, stromal cell 
decidualization has been induced in response to the expression of SOCS3, which was regulated by hormonal stimulation (83).

Jagged 1 (JAG1). JAG1 is the ligand for the receptor Notch1 and is involved in the biological processes of cell adhesion, motility, cell cycle regulation, cell communication and angiogenesis. Notch1 is expressed in endometrial epithelial and stromal cells, and mediates stromal differentiation and decidualization. Notch 1 and 2 are believed to be stem cell markers for ovarian cancer (84).

The present review shows that HNF-1 $\beta$ is involved in the differentiation program of tissue structures and tissue-specific lineage in several organs, including the ovaries, endometrium, liver, pancreas, kidneys and intestine.

\section{Summary}

CCC has several significant characteristics, based on morphological, behavioral and molecular features, which are distinct from those of other ovarian cancer histologies (85). CCC is the most common entity in Japan, accounting for $~ 70 \%$ of all endometriosis-associated ovarian cancers. The scope of computational technologies, including DNA microarrays, genome-wide gene expression profiling, microRNAs, methylation arrays and subsequent methods for the visualization of these datasets, has been extended to various transcriptomic and proteomic features that are focused on individual platforms (86).

The present review systematically identified and reevaluated the CCC-associated genes (Fig. 1). Firstly, due to advancement in computational predictions, HNF-1 $\beta$ has been unveiled as a major hub in the biology of CCC (91). With promoter hypomethylation, the expression of HNF-1 $\beta$ is significantly upregulated in endometriosis and endometriosis-associated ovarian CCC. HNF-1 $\beta$ is believed to be a master regulator of endodermal organogenesis and has long been discussed with regard to its role in clear cell carcinogenesis $(3-5,12,15,46,56,85-87)$. While the overexpression of HNF-1 $\beta$ may lead to cell regeneration, its potential role in malignant transformation has remained obscure. The present findings of this review highlight the importance of the HNF-1 $1 \beta$-induced global reproductive gene expression changes on morphology and ultimately on tissue/organ function. These changes included the process of the abnormal gain or loss of several significant genes, including decidualization, endometrial differentiation and regeneration, hormonal dependency, glycogen synthesis, detoxification, ion exchange and cell cycle regulation $(3,4,12,46,56,85)$. Collectively, the present review uncovers an unanticipated link between HNF-1 $\beta$ upregulation in CCC and the acquisition of cell cycle regulation under conditions of oxidative stress and inflammation. Cell cycle arrest may be a failsafe mechanism against the oxidative stress-induced DNA damage response (88).

Secondly, the present review demonstrated that HNF-1 $\beta$ is a strong inducer of endometrial receptivity, a morphogenetic program that is key to successful pregnancy. The endometrium undergoes morphological and functional changes during the menstrual cycle, which are essential for uterine receptivity $(12,56,88)$. These changes are driven by estrogen and progesterone and involve the fine control of numerous different genes, which have been induced by epigenetically-regulated HNF-1 $\beta$ (87). The specific expression of HNF-1 $\beta$ and its target genes has been confirmed in not only endometriosis and CCC, but also the secretory endometrium, the Arias-Stella glands and the decidua of the placenta (11). The Arias-Stella reaction may be the result of the regeneration and proliferation activity of the endometrial glands. A number of the HNF-1 $\beta$ target proteins may be hormonally-regulated and involved in the uteroplacental transport of substrates important in the implantation process and in early embryo-endometrial interactions $(46,56)$. The ectopic overexpression of HNF-1 $\beta$ in the normal secretory and gestational endometria may be sufficient to induce the morphological and molecular changes characteristic of a successful pregnancy. HNF-1 $\beta$ may be an activator to establish female reproduction, including secretory phase organogenesis, endometrial regeneration, differentiation, decidualization, glycogen synthesis, detoxification, cell cycle regulation, implantation, uterine receptivity, placentation and a successful pregnancy.

Thirdly, the overexpression of HNF-1 $\beta$ along with a lack of ER/PR expression may be used as a sensitive model system to identify the molecular characteristics of CCC. ER- $\alpha / E R-\beta$ and PRA/PRB are frequently expressed in ovarian cancer with a certain variability relating to histological subtype, grade and stage. In total, $70-100 \%$ of serous, mucinous and endometrioid cancers show positive nuclear staining for ER- $\alpha$. Conversely, CCC is negative for ER- $\alpha$ (89). ER- $\beta$ staining for CCC is similar to that of non-CCC. PRs have been detected in only $10 \%$ of CCC cases compared with non-CCC (80-90\%). DNA methylation and chromatin remodeling are two epigenetic mechanisms that have been linked with the lack of ER- $\alpha$ expression (90). These epigenetically modified genes may be involved in the expression of HNF-1 $\beta$ and hormone receptors.

Finally, cellular glycogen accumulation due to the promotion of glycogen synthesis is the most conspicuous feature of CCC (91). The regulated expression of the CCC-specific genes offers a mechanism to control the glycogen accumulation processes. HNF-1 $\beta$ regulates numerous aspects of glycogen synthesis and its target genes also delineate their interactions with signaling pathways in dictating glycogen synthesis. Glycogen is synthesized from glucose presumably to act as a source of energy for CCC cells and endometrial cells at implantation, placentation and successful pregnancy.

The present review expanded the repertoire of HNF-1 $\beta$ target genes, its downstream targets and its associated biological consequences, thereby shedding light on the complex regulatory circuitries of CCC. The evolutionarily conserved HNF family of transcription factors may play fundamental roles in regulating the hormonal microenvironment, secretory endometrial cell specification, glycogen synthesis and detoxification during pregnancy. The characteristics of two different entities, CCC cells and gestational endometrial cells (e.g. the Arias-Stella reaction), exhibit certain shared phenotypic and genetic features.

In conclusion, the results of the present review provide support for a marked degree of genetic overlap between CCC carcinogenesis and early gestational development.

\section{Acknowledgements}

This study was supported by a Grant-in-aid for Scientific Research from the Ministry of Education, Science and Culture of Japan granted to the Department of Obstetrics and Gynecology, Nara Medical University. 


\section{References}

1. Pectasides D, Pectasides E, Psyrri A and Economopoulos T: Treatment issues in clear cell carcinoma of the ovary: a different entity? Oncologist 11: 1089-1094, 2006.

2. Ozols RF, Bundy BN, Greer BE, Fowler JM, Clarke-Pearson D, Burger RA, Mannel RS, DeGeest K, Hartenbach EM and Baergen R; Gynecologic Oncology Group: Phase III trial of carboplatin and paclitaxel compared with cisplatin and paclitaxel in patients with optimally resected stage III ovarian cancer: a Gynecologic Oncology Group study. J Clin Oncol 21: 3194-3200, 2003.

3. Kajihara H, Yamada Y, Kanayama S, Furukawa N, Noguchi T, Haruta S, Yoshida S, Sado T, Oi H and Kobayashi H: Clear cell carcinoma of the ovary: potential pathogenic mechanisms (Review). Oncol Rep 23: 1193-1203, 2010.

4. Yamaguchi K, Mandai M, Oura T, Matsumura N, Hamanishi J, Baba T, Matsui S, Murphy SK and Konishi I: Identification of an ovarian clear cell carcinoma gene signature that reflects inherent disease biology and the carcinogenic processes. Oncogene 29 $1741-1752,2010$

5. Mandai M, Matsumura N, Baba T, Yamaguchi K, Hamanishi J and Konishi I: Ovarian clear cell carcinoma as a stress-responsive cancer: influence of the microenvironment on the carcinogenesis and cancer phenotype. Cancer Lett 310: 129-133, 2011.

6. Itamochi H, Kigawa J, Akeshima R, Sato S, Kamazawa S, Takahashi M, Kanamori Y, Suzuki M, Ohwada M and Terakawa N: Mechanisms of cisplatin resistance in clear cell carcinoma of the ovary. Oncology 62: 349-353, 2002.

7. Yoshida S, Furukawa N, Haruta S, Tanase Y, Kanayama S, Noguchi T, Sakata M, Yamada Y, Oi H and Kobayashi H: Theoretical model of treatment strategies for clear cell carcinoma of the ovary: focus on perspectives. Cancer Treat Rev 35: 608-615, 2009.

8. Higashiura Y, Kajihara H, Shigetomi $H$ and Kobayashi $H$ : Identification of multiple pathways involved in the malignant transformation of endometriosis (Review). Oncol Lett 4: 3-9, 2012.

9. Maeda D and Shih IeM: Pathogenesis and the role of ARID1A mutation in endometriosis-related ovarian neoplasms. Adv Anat Pathol 20: 45-52, 2013.

10. Toyokuni S. Mysterious link between iron overload and CDKN2A/2B. J Clin Biochem Nutr 48: 46-49, 2011.

11. Tanase Y, Yamada Y, Shigetomi H, Kajihara H, Oonogi A, Yoshizawa Y, Furukawa N, Haruta S, Yoshida S, Sado T, Oi H and Kobayashi H: Modulation of estrogenic action in clear cell carcinoma of the ovary. Exp Ther Med 3: 18-24, 2012.

12. Kajihara H, Yamada Y, Shigetomi H, Higashiura Y and Kobayashi $\mathrm{H}$ : The dichotomy in the histogenesis of endometriosis-associated ovarian cancer: clear cell-type versus endometrioid-type adenocarcinoma. Int J Gynecol Pathol 31: 304-312, 2012.

13. DeLair D, Oliva E, Köbel M, Macias A, Gilks CB and Soslow RA Morphologic spectrum of immunohistochemically characterized clear cell carcinoma of the ovary: a study of 155 cases. Am J Surg Pathol 35: 36-44, 2011

14. Hecht JL, Kotsopoulos J, Hankinson SE and Tworoger SS Relationship between epidemiologic risk factors and hormone receptor expression in ovarian cancer: results from the Nurses Health Study. Cancer Epidemiol Biomarkers Prev 18: 1624-3160, 2009.

15. Tsuchiya A, Sakamoto M, Yasuda J, Chuma M, Ohta T, Ohki M, Yasugi T, Taketani Y and Hirohashi S: Expression profiling in ovarian clear cell carcinoma: identification of hepatocyte nuclear factor-1 beta as a molecular marker and a possible molecular target for therapy of ovarian clear cell carcinoma. Am J Pathol 163: 2503-2512, 2003

16. Uchida H, Maruyama T, Nishikawa-Uchida S, Oda H, Miyazaki K, Yamasaki A and Yoshimura Y: Studies using an in vitro model show evidence of involvement of epithelial-mesenchymal transition of human endometrial epithelial cells in human embryo implantation. J Biol Chem 287: 4441-4450, 2012.

17. Wareing $M$ and Greenwood SL: Review: Potassium channels in the human fetoplacental vasculature. Placenta 32: S203-S206, 2011.

18. Milne SA, Henderson TA, Kelly RW, Saunders PT, Baird DT and Critchley HO: Leukocyte populations and steroid receptor expression in human first-trimester decidua; regulation by antiprogestin and prostaglandin E analog. J Clin Endocrinol Metab 90: 4315-4321, 2005.
19. Bulun SE, Cheng YH, Pavone ME, Xue Q, Attar E, Trukhacheva E, Tokunaga $H$, Utsunomiya $H$, Yin $P$, Luo $X$, Lin Z, Imir G, Thung S, Su EJ and Kim JJ: Estrogen receptor-beta, estrogen receptor-alpha, and progesterone resistance in endometriosis. Semin Reprod Med 28: 36-43, 2010.

20. Akahane T, Sekizawa A, Okuda T, Kushima M, Saito H and Okai T: Disappearance of steroid hormone dependency during malignant transformation of ovarian clear cell cancer. Int J Gynecol Pathol 24: 369-376, 2005.

21. Dhingra N, Punia RS, Radotra A and Mohan H: Arias-Stella reaction in upper genital tract in pregnant and non-pregnant women: a study of 120 randomly selected cases. Arch Gynecol Obstet 276: 47-52, 2007

22. Trpkov K, Guggisberg K and Yilmaz A: Arias-Stella reaction as a diagnostic pitfall in a bladder biopsy with endometriosis: case report and review of the pseudoneoplastic bladder lesions. Pathol Res Pract 205: 653-656, 2009.

23. Sakaki M, Hirokawa M, Sano T, Takahashi H, Tezuka K, Abe K and Sano M: Ovarian endometriosis showing decidual change and Arias-Stella reaction with biotin-containing intranuclear inclusions. Acta Cytol 47: 321-324, 2003.

24. Ohkawa K, Amasaki H, Terashima Y, Aizawa S and Ishikawa E: Clear cell carcinoma of the ovary: light and electron microscopic studies. Cancer 40: 3019-3029, 1977.

25. Kato $\mathrm{N}$ and Motoyama T: Overexpression of osteopontin in clear cell carcinoma of the ovary: close association with HNF-1beta expression. Histopathology 52: 682-688, 2008.

26. Matsuura M, Suzuki T and Saito T: Osteopontin is a new target molecule for ovarian clear cell carcinoma therapy. Cancer Sci 101: 1828-1833, 2010.

27. Fraga-Silva RA, Costa-Fraga FP, Murça TM, et al: Angiotensinconverting enzyme 2 activation improves endothelial function. Hypertension 61: 1233-1238, 2013.

28. Min KW, Park MH, Hong SR, Lee H, Kwon SY, Hong SH, Joo HJ, Park IA, An HJ, Suh KS, Oh HK, Yoo CW, Kim MJ, Chang HK, Jun SY, Yoon HK, Chang ED, Kim DW and Kim I; The Gynecologic Pathology Study Group of the Korean Society of Pathologists: Clear Cell Carcinomas of the Ovary: A Multi-Institutional Study of 129 Cases in Korea With Prognostic Significance of Emil and Galectin-3. Int J Gynecol Pathol 32: 3-14, 2013.

29. Oishi T, Itamochi H, Kigawa J, Kanamori Y, Shimada M, Takahashi M, Shimogai R, Kawaguchi W, Sato S and Terakawa N: Galectin-3 may contribute to Cisplatin resistance in clear cell carcinoma of the ovary. Int J Gynecol Cancer 17: 1040-1046, 2007

30. von Wolff M, Wang X, Gabius HJ and Strowitzki T: Galectin fingerprinting in human endometrium and decidua during the menstrual cycle and in early gestation. Mol Hum Reprod 11: 189-194, 2005.

31. Noël JC, Chapron C, Borghese B, Fayt I and Anaf V: Galectin-3 is overexpressed in various forms of endometriosis. Appl Immunohistochem Mol Morphol 19: 253-257, 2011.

32. Maeda D, Ota S, Takazawa Y, Aburatani H, Nakagawa S, Yano T, Taketani Y, Kodama T and Fukayama M: Glypican-3 expression in clear cell adenocarcinoma of the ovary. Mod Pathol 22: 824-832, 2009.

33. Tamada Y, Takeuchi H, Suzuki N, Susumu N, Aoki D and Irimura T: Biological and therapeutic significance of MUC1 with sialoglycans in clear cell adenocarcinoma of the ovary. Cancer Sci 98: 1586-1591, 2007.

34. Vlad AM, Diaconu I and Gantt KR: MUC1 in endometriosis and ovarian cancer. Immunol Res 36: 229-236, 2006.

35. Carson DD, Julian J, Lessey BA, Prakobphol A and Fisher SJ: MUC1 is a scaffold for selectin ligands in the human uterus. Front Biosci 11: 2903-2908, 2006.

36. Kim A, Enomoto T, Serada S, Ueda Y, Takahashi T, Ripley B, Miyatake T, Fujita M, Lee CM, Morimoto K, Fujimoto M, Kimura $\mathrm{T}$ and Naka T: Enhanced expression of Annexin A4 in clear cell carcinoma of the ovary and its association with chemoresistance to carboplatin. Int J Cancer 125: 2316-2322, 2009.

37. Ponnampalam AP and Rogers PA: Cyclic changes and hormonal regulation of annexin IV $\mathrm{mRNA}$ and protein in human endometrium. Mol Hum Reprod 12: 661-669, 2006

38. Jiang YL, Li B, Xing FQ, Wang F and Feng JH: Study on the relationship between altered expression of annexin $\mathrm{A} 4$ and endometrial receptivity during the implantation window in infertile patients with endometriosis. Zhonghua Fu Chan Ke Za Zhi 47: 324-327, 2012 (In Chinese). 
39. Sugita S, Morishita Y, Kano J, Furuya S, Shiba-Ishii A and Noguchi M: IGFBP-1 is expressed specifically in ovarian clear cell adenocarcinoma. Histopathology 58: 729-738, 2011.

40. Tan DS, Iravani M, McCluggage WG, Lambros MB, Milanezi F, Mackay A, Gourley C, Geyer FC, Vatcheva R, Millar J, Thomas K, Natrajan R, Savage K, Fenwick K, Williams A, Jameson C, El-Bahrawy M, Gore ME, Gabra H, Kaye SB, Ashworth A and Reis-Filho JS: Genomic analysis reveals the molecular heterogeneity of ovarian clear cell carcinomas. Clin Cancer Res 17: 1521-1534, 2011.

41. Howell NR, Zheng W, Cheng L, Tornos C, Kane P, Pearl M, Chalas E and Liang SX: Carcinomas of ovary and lung with clear cell features: can immunohistochemistry help in differential diagnosis? Int J Gynecol Pathol 26: 134-140, 2007.

42. Pacchiarotti A, Caserta D, Sbracia M and Moscarini M: Expression of oct- 4 and c-kit antigens in endometriosis. Fertil Steril 95: 1171-1173, 2011

43. Ghosh D and Sengupta J: Endocrine and paracrine correlates of endometrial receptivity to blastocyst implantation in the human. Indian J Physiol Pharmacol 48: 6-30, 2004

44. Li B, Jin H, Yu Y, Gu C, Zhou X, Zhao N and Feng Y: HOXA10 is overexpressed in human ovarian clear cell adenocarcinoma and correlates with poor survival. Int J Gynecol Cancer 19: $1347-1352,2009$

45. Zanatta A, Rocha AM, Carvalho FM, Pereira RM, Taylor HS, Motta EL, Baracat EC and Serafini PC: The role of the Hoxa10/ HOXA10 gene in the etiology of endometriosis and its related infertility: a review. J Assist Reprod Genet 27: 701-710, 2010

46. Kobayashi H, Yamada Y, Kanayama S, Furukawa N, Noguchi T, Haruta S, Yoshida S, Sakata M, Sado T and Oi H: The role of hepatocyte nuclear factor-1beta in the pathogenesis of clear cell carcinoma of the ovary. Int J Gynecol Cancer 19: 471-479, 2009

47. Salameh W, Helliwell JP, Han G, McPhaul L and Khorram O Expression of endometrial glycogen synthase kinase-3beta protein throughout the menstrual cycle and its regulation by progesterone. Mol Hum Reprod 12: 543-549, 2006.

48. von Wolff M, Ursel S, Hahn U, Steldinger R and Strowitzki T: Glucose transporter proteins (GLUT) in human endometrium: expression, regulation, and function throughout the menstrua cycle and in early pregnancy. J Clin Endocrinol Metab 88: 3885-3892, 2003.

49. Senkel S, Lucas B, Klein-Hitpass L and Ryffel GU: Identification of target genes of the transcription factor HNF1beta and HNF1alpha in a human embryonic kidney cell line. Biochim Biophys Acta 1731: 179-190, 2005.

50. Xu JM, Wang Y, Ge FJ, Lin L, Liu ZY and Sharma MR: Severe irinotecan-induced toxicity in a patient with UGT1A $1 * 28$ and UGT1A1*6 polymorphisms. World J Gastroenterol 19: 3899-3903, 2013

51. Tang SW, Yang TC, Lin WC, Chang WH, Wang CC, Lai MK and Lin JY: Nicotinamide N-methyltransferase induces cellular invasion through activating matrix metalloproteinase-2 expression in clear cell renal cell carcinoma cells. Carcinogenesis 32: 138-145, 2011.

52. Salleh N, Baines DL, Naftalin RJ and Milligan SR: The hormonal control of uterine luminal fluid secretion and absorption. J Membr Biol 206: 17-28, 2005.

53. Kerschner JL, Harris A. Transcriptional networks driving enhancer function in the CFTR gene. Biochem J 446: 203-212, 2012.

54. Ferrè S, Veenstra GJ, Bouwmeester R, Hoenderop JG, Bindels RJ. HNF-1B specifically regulates the transcription of the $\gamma$ a-subunit of the Na+/K+-ATPase. Biochem Biophys Res Commun 404: 284-290, 2011

55. Kobayashi H, Kajiwara H, Kanayama S, Yamada Y, Furukawa N, Noguchi T, Haruta S, Yoshida S, Sakata M, Sado T, Oi H. Molecular pathogenesis of endometriosis-associated clear cell carcinoma of the ovary (Review). Oncol Rep 22: 233-240, 2009.

56. Gütgemann I, Lehman NL, Jackson PK and Longacre TA: Emil protein accumulation implicates misregulation of the anaphase promoting complex/cyclosome pathway in ovarian clear cell carcinoma. Mod Pathol 21: 445-454, 2008.

57. Kuo KT, Mao TL, Chen X, Feng Y, Nakayama K, Wang Y, Glas R, Ma MJ, Kurman RJ, Shih IeM and Wang TL: DNA copy numbers profiles in affinity-purified ovarian clear cell carcinoma. Clin Cancer Res 16: 1997-2008, 2010.

58. Ju W, Yoo BC, Kim IJ, Kim JW, Kim SC and Lee HP: Identification of genes with differential expression in chemoresistant epithelial ovarian cancer using high-density oligonucleotide microarrays. Oncol Res 18: 47-56, 2009.
59. Ozdemir F, Altinisik J, Karateke A, Coksuer H and Buyru N: Methylation of tumor suppressor genes in ovarian cancer. Exp Ther Med 4: 1092-1096, 2012.

60. Ho CM, Lai HC, Huang SH, Chien TY, Lin MC and Chang SF: Promoter methylation of sFRP5 in patients with ovarian clear cell adenocarcinoma. Eur J Clin Invest 40: 310-318, 2010.

61. Akatsuka S, Yamashita Y, Ohara H, Liu YT, Izumiya M, Abe K, Ochiai M, Jiang L, Nagai H, Okazaki Y, Murakami H, Sekido Y, Arai E, Kanai Y, Hino O, Takahashi T, Nakagama H and Toyokuni S: Fenton reaction induced cancer in wild type rats recapitulates genomic alterations observed in human cancer. PLoS One 7: e43403, 2012.

62. Choi YL, Kang SY, Shin YK, Choi JS, Kim SH, Lee SJ, Bae DS and Ahn G: Aberrant hypermethylation of RASSF1A promoter in ovarian borderline tumors and carcinomas. Virchows Arch 448 : 331-336, 2006

63. Vestergaard AL, Thorup K, Knudsen UB, Munk T, Rosbach H, Poulsen JB, Guldberg P and Martensen PM: Oncogenic events associated with endometrial and ovarian cancers are rare in endometriosis. Mol Hum Reprod 17: 758-761, 2011.

64. Zannoni GF, Morassi F, Prisco MG, De Stefano I, Vellone VG, Arena V, Scambia G and Gallo D: Clinicopathologic and immunohistochemical features of ovarian clear cell carcinomas in comparison with type I and type II tumors. Int J Gynecol Pathol 31: 507-516, 2012.

65. Eltabbakh GH, Mount SL, Beatty B, Simmons-Arnold L and Cooper K: Clinical and molecular differences between clear cell and papillary serous ovarian carcinoma. J Surg Oncol 93: 379-386, 2006

66. Axiotis CA, Guarch R, Merino MJ, Laporte N and Neumann RD P-glycoprotein expression is increased in human secretory and gestational endometrium. Lab Invest 65: 577-581, 1991.

67. Nomura H, Tamada Y, Miyagi T, Suzuki A, Taira M, Suzuki N, Susumu N, Irimura T and Aoki D: Expression of NEU3 (plasma membrane-associated sialidase) in clear cell adenocarcinoma of the ovary: its relationship with T factor of pTNM classification. Oncol Res 16: 289-297, 2006

68. Liu P, Khurana A, Rattan R, He X, Kalloger S, Dowdy S, Gilks B and Shridhar V: Regulation of HSulf-1 expression by variant hepatic nuclear factor 1 in ovarian cancer. Cancer Res 69: 4843-4850, 2009.

69. Collins Y, Dicioccio R, Keitz B, Lele S and Odunsi K: Methylation of death-associated protein kinase in ovarian carcinomas. Int J Gynecol Cancer 16: 195-199, 2006

70. Song H, Kwan SY, Izaguirre DI, Zu Z, Tsang YT, Tung CS King ER, Mok SC, Gershenson DM and Wong KK: PAX2 Expression in Ovarian Cancer. Int J Mol Sci 14: 6090-6105, 2013.

71. Cai KQ, Caslini C, Capo-chichi CD, Slater C, Smith ER, Wu H, Klein-Szanto AJ, Godwin AK and Xu XX: Loss of GATA4 and GATA6 expression specifies ovarian cancer histological subtypes and precedes neoplastic transformation of ovarian surface epithelia. PLoS One 4: e6454, 2009.

72. Blanco D, Vicent S, Fraga MF, Fernandez-Garcia I, Freire J, Lujambio A, Esteller M, Ortiz-de-Solorzano C, Pio R, Lecanda F and Montuenga LM: Molecular analysis of a multistep lung cancer model induced by chronic inflammation reveals epigenetic regulation of p16 and activation of the DNA damage response pathway. Neoplasia 9: 840-852, 2007.

73. McKie AB, Vaughan S, Zanini E, Okon IS, Louis L, de Sousa C, Greene MI, Wang Q, Agarwal R, Shaposhnikov D, Wong JL, Gungor H, Janczar S, El-Bahrawy M, Lam EW, Chayen NE and Gabra H: The OPCML tumor suppressor functions as a cell surface repressor-adaptor, negatively regulating receptor tyrosine kinases in epithelial ovarian cancer. Cancer Discov 2: 156-171, 2012.

74. Ren F, Wang D, Jiang Y and Ren F: Epigenetic inactivation of hMLH1 in the malignant transformation of ovarian endometriosis. Arch Gynecol Obstet 285: 215-221, 2012.

75. Coffinier C, Thepot D, Babinet C, Yaniv M and Barra J: Essential role for the homeoprotein $\mathrm{vHNF} 1 / \mathrm{HNF} 1$ beta in visceral endoderm differentiation. Development 126: 4785-4794, 1999.

76. Horikawa Y, Iwasaki N, Hara M, Furuta H, Hinokio Y, Cockburn BN, Lindner T, Yamagata K, Ogata M, Tomonaga $\mathrm{O}$, Kuroki H, Kasahara T, Iwamoto $\mathrm{Y}$ and Bell GI: Mutation in hepatocyte nuclear factor- $1 \beta$ gene (TCF2) associated with MODY. Nat Genet 17: 384-385, 1997.

77. Ogata K, Shimamura Y, Hamada K, Hisa M, Bun M, Okada N, Inoue $\mathrm{K}$, Taniguchi $\mathrm{Y}$, Ishihara M, Kagawa $\mathrm{T}$, Horino $\mathrm{T}$, Fujimoto $S$ and Terada Y: Upregulation of HNF-1 $\beta$ during experimental acute kidney injury plays a crucial role in renal tubule regeneration. Am J Physiol Renal Physiol 303: F689-F699, 2012. 
78. D'Angelo A, Bluteau O, Garcia-Gonzalez MA, Gresh L, Doyen A, Garbay S, Robine S and Pontoglio M: Hepatocyte nuclear factor 1alpha and beta control terminal differentiation and cell fate commitment in the gut epithelium. Development 137: 1573-1582, 2010.

79. Avni FE and Hall M: Renal cystic diseases in children: new concepts. Pediatr Radiol 40: 939-946, 2010.

80. Zhou XH, Hui ZY and Li Y: Clinical and pathological features of a neonate with autosomal recessive polycystic kidney disease caused by a nonsense PKHD1 mutation. World J Pediatr 9: 76-79, 2013.

81. Serafini-Cessi F, Monti A and Cavallone D: N-Glycans carried by Tamm-Horsfall glycoprotein have a crucial role in the defense against urinary tract diseases. Glycoconj J 22: 383-394, 2005.

82. Sutherland KD, Lindeman GJ, Choong DY, Wittlin S, Brentzell L, Phillips W, Campbell IG and Visvader JE: Differential hypermethylation of SOCS genes in ovarian and breast carcinomas. Oncogene 23: 7726-7733, 2004.

83. Dimitriadis E, Stoikos C, Tan YL and Salamonsen LA Interleukin 11 signaling components signal transducer and activator of transcription 3 (STAT3) and suppressor of cytokine signaling 3 (SOCS3) regulate human endometrial stromal cell differentiation. Endocrinol 147: 3809-3817, 2006.

84. Oktem G, Sanci M, Bilir A, Yildirim Y, Kececi SD, Ayla S and Inan S: Cancer stem cell and embryonic development-associated molecules contribute to prognostic significance in ovarian cancer. Int J Gynecol Cancer 22: 23-29, 2012.
85. Yamada Y, Shigetomi H, Onogi A, Haruta S, Kawaguchi R, Yoshida S, Furukawa N, Nagai A, Tanase Y, Tsunemi T, Oi H and Kobayashi H: Redox-active iron-induced oxidative stress in the pathogenesis of clear cell carcinoma of the ovary. Int J Gynecol Cancer 21: 1200-1207, 2011.

86. Haruta S, Furukawa N, Yoshizawa Y, Tsunemi T, Nagai A, Kawaguchi R, Tanase Y, Yoshida S and Kobayashi H: Molecular genetics and epidemiology of epithelial ovarian cancer. Oncol Rep 26: 1347-1356, 2011.

87. Kato N, Tamura G and Motoyama T: Hypomethylation of hepatocyte nuclear factor-1beta (HNF-1beta) CpG island in clear cell carcinoma of the ovary. Virchows Arch 452: 175-180, 2008.

88. Shigetomi H, Higashiura Y, Kajihara H and Kobayashi H: A potential link of oxidative stress and cell cycle regulation for development of endometriosis. Gynecol Endocrinol 28: 897-902, 2012.

89. Cameron RI, Ashe P, O'Rourke DM, Foster H and McCluggage WG: A panel of immunohistochemical stains assists in the distinction between ovarian and renal clear cell carcinoma. Int J Gynecol Pathol 22: 272-276, 2003.

90. Brinkman JA and El-Ashry D: ER re-expression and re-sensitization to endocrine therapies in ER-negative breast cancers. J Mammary Gland Biol Neoplasia 14: 67-78, 2009.

91. Iida Y, Aoki K, Asakura T, Ueda K, Yanaihara N, Takakura S, Yamada K, Okamoto A, Tanaka T and Ohkawa K: Hypoxia promotes glycogen synthesis and accumulation in human ovarian clear cell carcinoma. Int J Oncol 40: 2122-2130, 2012. 